\title{
A New Generalised Approach for Feature Representation in Vibroacoustical Fault Diagnosis
}

\author{
L. Gelman ${ }^{\dagger}$, P. Jenkin, M. Sanderson and C. Thompson \\ Cranfield University, Cranfield, Bedfordshire MK43 OAL, UK \\ M.J. Crocker ${ }^{\dagger}$ \\ Department of Mechanical Engineering, 202 Ross Hall, Auburn University, AL 36849, USA
}

(Received 30 July 2003; accepted 7 December 2003)

\begin{abstract}
A new generalised approach for health monitoring has been formulated. The generalised approach consists of using two new monitoring features simultaneously, which are based on the Fourier transform and which take into account the covariance between features. The generalisation of the approach improves the effectiveness of health monitoring. The monitoring effectiveness between the generalised approach and the power spectral density approach was compared for vibroacoustical monitoring. It was found that the power spectral density approach is not optimal, and represents only a particular case of the generalised approach. The power spectral density approach is optimal only if both: the correlation coefficient between new features is equal to zero, and the standard deviations of the features are equal. Use of the generalised approach provides an improvement in monitoring effectiveness as compared with the power spectral density approach. Application of the generalised approach was considered for the vibroacoustical diagnostics of damping.
\end{abstract}

${ }^{\dagger}$ Member of the International Institute of Acoustics and Vibration (IIAV)

\section{INTRODUCTION}

A new generic approach has been proposed for those cases in which one- or multidimensional Fourier transforms are used for health monitoring and pattern recognition. ${ }^{1}$ The approach consists of using two new monitoring features simultaneously: the real and the imaginary components of the Fourier transform. It was shown that the approach is more general than the power spectral density (PSD), phase spectrum, and Hartley approaches and is more effective than the PSD approach. ${ }^{1-3}$

This is in contrast to other condition monitoring and pattern recognition applications, including Fourier optics applications, where the PSD or phase spectrum are used. . $^{4-23}$

However, the approach has been proposed and investigated only for one particular case, without taking into account the covariance between new monitoring features. ${ }^{1-3}$ Taking covariance between monitoring features into account may improve the monitoring effectiveness. Therefore to generalise the proposed approach, we need to take into account the covariance between the features, i.e., Fourier components. Application of the generalised approach to a particular health monitoring task was not undertaken as well.

The purposes of this technical note are:

- to generalise the approach ${ }^{1}$ and estimate the effect of generalisation;

- to estimate and compare the monitoring effectiveness of the generalised approach with the monitoring effectiveness of the PSD approach; and

- to employ the generalised approach for the acoustical monitoring of damping.

\section{THEORETICAL ANALYSIS}

The approach ${ }^{1}$ consists of using simultaneously the real $X_{R}$ and imaginary $X_{I}$ components of the Fourier transform at the frequency $\omega_{x}$ as monitoring features:

$$
\begin{aligned}
& X_{R}\left(\omega_{x}, T\right)=\int_{0}^{T} x(t) \cos \omega_{x} t d t ; \\
& X_{I}\left(\omega_{x}, T\right)=\int_{0}^{T} x(t) \sin \omega_{x} t d t,
\end{aligned}
$$

where $T$ is the duration of the rectangular window of the shorttime Fourier transform.

We consider the general case of the short-time Fourier transform $(T \neq \infty)$. Generally, for the short-time Fourier transform the correlation coefficient between the features Eqs. (1) and (2) are nonzero.

Therefore we generalise the approach. ${ }^{1}$ The generalised approach consists of using two new recognition features simultaneously: the real and imaginary components of the Fourier transform, taking into account the covariance between these features (instead of the usual correlation coefficient for the time series $x(t)$ ).

Now we employ the generalised approach for two-class monitoring of the Gaussian narrowband-centred vibroacoustical signals $x(t)$ with different variances $\sigma_{x j}^{2}$ (e.g. $\sigma_{x 0} \neq \sigma_{x 1}$ ) and identical normalised autocovariance functions $r_{x 0}=r_{x 1}$ $=r_{x}$ for classes $H_{j}, j=0,1$. The monitoring information is contained in the short-time Fourier transform for the fre- 